\title{
Estudio exploratorio sobre el uso de instrumentos de evaluación cognitiva y neuropsicológica en centros de personas mayores de
} Galicia

\author{
David Facal $^{\mathrm{a}, \mathrm{f}}$, Romina Mouriz Corbelle ${ }^{\mathrm{b}, \mathrm{f}}$, Aranzazu Balo-García ${ }^{\mathrm{c}, \mathrm{d}, \mathrm{f}}$, Isabel González- \\ Abraldes $^{\mathrm{c}, \mathrm{d}, \mathrm{f}}$, Xosé Caamaño Ponte ${ }^{\mathrm{e}, \mathrm{f}}$, Carlos Dosil Díaz ${ }^{\mathrm{a}, \mathrm{b}, \mathrm{f}}$ y José C. Millán-Calenti ${ }^{\mathrm{c}, \mathrm{d}}$ \\ ${ }^{a}$ Departamento de Psicoloxía Evolutiva e da Educación, Universidade de Santiago de Compostela, Santiago de Compostela, \\ España \\ ${ }^{b}$ Centro Xerontológico A Veiga, Lugo, España \\ ${ }^{c}$ Centro Gerontológico La Milagrosa, A Coruña, España \\ ${ }^{d}$ Grupo de Investigación en Gerontología, Universidade de A Coruña, A Coruña, España \\ e Mayores Atención a la Dependencia, A Coruña, España \\ ${ }^{f}$ Grupo de Traballo sobre Avaliación Cognitiva e Neuropsicolóxica, Sociedade Galega de Xerontoloxía e Xeriatría (SGXX), España
}

\section{Resumen}

Introducción: La evaluación cognitiva es una herramienta fundamental en los centros de mayores a la hora de planificar la atención e individualizar la intervención. La Sociedade Galega de Xerontoloxía e Xeriatría ha constituido un Grupo de Trabajo sobre Evaluación Cognitiva y Neuropsicológica con el objetivo de estudiar los instrumentos empleados e identificar los principales retos en esta área.

Material y métodos: Se realizó un estudio exploratorio a través de encuesta en línea a los socios de la Sociedade Galega de Xerontoloxía e Xeriatría entre diciembre de 2013 y enero de 2014. Participaron 49 profesionales, de los cuales 26 eran profesionales de la psicología y 10 de la medicina.

Resultados: Se encuentra un consenso en el uso del MMSE como instrumento de cribado y del GDS como escala global de la demencia. Existe mayor variabilidad en las baterías generales para la valoración de las demencias (CAMCOG-R, ADAS-Cog, Test Barcelona abreviado) y en el uso de pruebas específicas y de baterías de deterioro severo.

Conclusiones: Se sugiere un riesgo de polarización del sector, en el que un porcentaje elevado de profesionales emplearía unos pocos instrumentos generales y un porcentaje pequeño un número elevado de instrumentos específicos. Se subraya la necesidad de un consenso que facilite el desempeño, objetive las necesidades formativas, maximice la relación entre evaluación e intervención cognitiva y proporcione herramientas para valorar el cambio.

Palabras clave: Evaluación cognitiva, Evaluación neuropsicológica, Tests de cribado, Escalas globales de demencia, Baterías de evaluación del deterioro severo

An exploratory study on the use of cognitive and neuropsychological assessessments in centres for the elderly in Galicia

\begin{abstract}
Introduction: Cognitive assessment is a fundamental tool in centres for the elderly when planning individualized care and intervention. The Galician Society of Gerontology and Geriatrics (Sociedade Galega of Xerontoloxía and Xeriatría) has formed a Working Group on Cognitive and Neuropsychological Assessment, with the aim of studying the tools used and to identify the main challenges in this area.

Material and methods: An exploratory study was conducted via an online questionnaire with Sociedade Galega of Xerontoloxía and Xeriatría members between December 2013 and January 2014. It was completed by 49 professionals, of whom 26 were professionals in psychology and 10 medical doctors.

Results: Consensus was found on the use of the MMSE as a screening tool, as well as the Global Deterioration Scale for dementia. There is a wide variation in the choice of general batteries of tests for the assessment of dementia (CAMCOG-R, ADAS-Cog, abbreviated Barcelona Test), and of the use of specific tests and a severe impairment battery.

Conclusions: The risk of bias in the sector is suggested, with a high percentage of general practitioners using a few tools, and a small percentage using a large number of specific tools. The need for a consensus is stressed in order to
\end{abstract}


facilitate assessment, objectify training needs, maximize the relationship between assessment and cognitive intervention, and provide tools to assess change.

Keywords: Cognitive assessment, Neuropsychological assessment, Screening tests, Global dementia scales, Severe impairment batteries

\section{Introducción}

La evaluación cognitiva y neuropsicológica es una tarea central en los diferentes dispositivos y centros de atención a las personas mayores de cara al diagnóstico de alteraciones cognitivas, la identificación de patrones de funcionamiento y el desarrollo de estrategias de intervención y manejo ${ }^{1}$. Respecto a la evaluación para la detección precoz del deterioro cognitivo, los beneficios de un diagnóstico precoz incluyen el confirmar las sospechas de deterioro cognitivo, promover estrategias de afrontamiento y facilitar la planificación personal. Respecto a la identificación de patrones de funciones, si bien el diagnóstico de demencia es clínico, la valoración cognitiva desempeña un rol crítico en la identificación de la severidad de la alteración de determinadas áreas intelectuales ${ }^{2}$. Un objetivo secundario de la evaluación cognitiva es establecer una línea base de funcionamiento cognitivo que pueda ser usada para determinar la eficacia de las intervenciones ${ }^{1,3}$.

Aunque disponemos de diferentes tipos de evaluación cognitiva según sus objetivos, extensión y dificultad (breve o de cribado cognitivo, extensa o completa, y específica de determinadas funciones cognitivas orientada a la intervención), teniendo en cuenta la carencia en la práctica diaria de tiempo real por parte de los profesionales que realizan su actividad en los centros de personas mayores, surge la necesidad de desarrollar instrumentos sencillos y rápidos que permitan detectar en unos minutos a aquellas personas que puedan padecer una demencia ${ }^{4}$. Las pruebas de cribado deberían ser sensibles en etapas iniciales del deterioro cognitivo e incluir, al menos, una evaluación de la memoria episódica y funciones ejecutivas, ya que son los dominios más alterados en la enfermedad de Alzheimer y la demencia vascular, las causas más frecuentes de demencia ${ }^{5}$ (tabla 1 ).

Una vez establecida la sospecha de manera objetiva de deterioro cognitivo pasaremos al empleo de pruebas específicas para la valoración de las demencias ${ }^{6}$ como la Escala de la Evaluación de la Enfermedad de Alzheimer - Subescala Cognitiva (ADAS-COG) y el Examen Cognitivo de Cambridge Revisado (CAMCOG-R), o en nuestro contexto la versión abreviada del Test Barcelona (tabla 1). Estos test han sido diseñados para cuantificar determinadas funciones cognitivas, es decir, no establecen un diagnóstico, sino que cuantifican la severidad de la alteración de determinadas áreas intelectuales. Las escalas de graduación clínica, como Clinical Dementia Rating Scale (CDR), la Global Deterioration Scale (GDS) o el Functional Assessment Staging (FAST) (tabla 1) ${ }^{8}$, tienen por objeto evaluar el cambio clínicamente significativo sobre la base de la evaluación multidimensional, teniendo en cuenta la evaluación de la cognición, el comportamiento y la capacidad funcional ${ }^{7}$.

Las escalas de graduación clínica, aunque son eficaces ofreciendo una descripción de las áreas de deterioro cognitivo, no ofrecen la suficiente información sobre el estado cognitivo del paciente para poder realizar un adecuado plan de intervención. Por este motivo, surge la necesidad de utilizar escalas más específicas para la valoración del deterioro en diferentes áreas cognitivas (lenguaje, percepción y atención, memoria, funciones ejecutivas), (tabla 1). Respecto a la evaluación específica del estado cognitivo de los usuarios con deterioro avanzado, la realidad es que existen, al menos en castellano, pocos instrumentos, y que estos tienen limitaciones a la hora de ser aplicados. A la hora de valorar a usuarios con deterioro cognitivo en estadio moderado-grave es importante centrarse en capacidades y funciones que aún conserva en su vida diaria, y completar la información a través de los cuidadores informales y/o

formales ${ }^{1}$.

Dada la diversidad de instrumentos de evaluación disponibles, y a la presencia de retos prácticos en la aplicación de estas pruebas (adecuada detección precoz del deterioro cognitivo, adecuación entre evaluación e intervención, evaluación del cambio y del efecto de las intervenciones, problemas de validez ecológica, bajo nivel socioeducativo de las personas mayores en nuestro contexto), la Sociedade Galega de Xerontoloxía e Xeriatría (SGXX) constituyó un Grupo de Trabajo sobre Evaluación Cognitiva y Neuropsicológica en centros de personas mayores. Dicho grupo ha desarrollado el estudio exploratorio que presentamos, con una doble finalidad: a) estudiar qué instrumentos de evaluación cognitiva y neuropsicológica se aplican en los centros de mayores de Galicia, y b) recoger los intereses y dificultades de los profesionales gallegos con respecto a la evaluación cognitiva. En base a ello, el objetivo principal del presente trabajo es sintetizar los principales hallazgos de dicho estudio exploratorio, presentando los instrumentos de uso más común en centros gallegos y planteando una serie de retos que permitan mejorar 
en la detección, clasificación y atención a las personas en el continuum declive cognitivo asociado a la edad vs. demencia.

\section{Material y métodos}

\section{Procedimiento y materiales}

El Grupo de Trabajo sobre Evaluación Cognitiva y Neuropsicológica de la SGXX elaboró un cuestionario sobre los instrumentos de evaluación cognitiva y neuropsicológica empleados (Anexo) por los diferentes profesionales. Se trata de un cuestionario breve de respuesta múltiple en el que se preguntaba a los profesionales acerca de los test empleados en la valoración cognitiva (pruebas de cribado, escalas globales de demencia, baterías neuropsicológicas, pruebas de deterioro severo y test específicos de funciones cognitivas) ${ }^{9}$. Se incluyeron además una serie de preguntas abiertas, para que los profesionales opinaran acerca de las principales limitaciones a la hora de aplicar las pruebas, si disponían de formación específica sobre evaluación cognitiva y neuropsicológica y por último sobre la importancia que tenía en el funcionamiento de su centro de trabajo la evaluación cognitiva. El cuestionario se configuró planteando interrogantes relacionados con aspectos clave de la valoración cognitiva, teniendo en cuenta tanto la multitud de instrumentos disponibles como la diversidad de los recursos de atención dirigidos a las personas mayores en Galicia. En este sentido, se ha tenido en cuenta la heterogeneidad en cuanto al perfil de profesionales y la heterogeneidad en la aplicación, incluida la disponibilidad de herramientas de evaluación pertenecientes a contextos distintos al institucional.

El cuestionario se implementó en la página web de la SGXX (www.sgxx.es) para a continuación establecer contacto vía mail a través de la lista de distribución de la Sociedad, invitando a todos los socios a participar en el estudio cumplimentando el cuestionario, que estuvo disponible durante los meses de diciembre de 2013 y enero de 2014. Las respuestas fueron registradas a través del servidor de la SGXX y volcadas a un documento de Excel para su posterior análisis. Para las respuestas de tipo cualitativo, se realizó un análisis de contenido.

Tabla 1

Instrumentos de evaluación cognitiva y neuropsicológica de uso común en gerontología

\begin{tabular}{|c|c|c|c|}
\hline Tipología & Instrumento & Descripción & Referencia/s \\
\hline \multirow[t]{3}{*}{$\begin{array}{l}\text { Instrumentos } \\
\text { de cribado }\end{array}$} & $\begin{array}{l}\text { Mini - Mental State } \\
\text { Examination (MMSE) }\end{array}$ & $\begin{array}{l}\text { Escala cognitiva abreviada con amplia validez } \\
\text { y difusión internacional. El uso repetido no } \\
\text { tiene efectos de práctica y es sensible con } \\
\text { poblaciones de riesgo asociado a la edad. Entre } \\
\text { sus limitaciones destaca la falta de sensibilidad } \\
\text { para detectar fases iniciales de demencia, ya } \\
\text { que apenas evalúa funciones ejecutivas, y el } \\
\text { efecto del nivel educativo }\end{array}$ & $\begin{array}{l}\text { - Lobo A, Saz P, Marcos G, Día JL, de } \\
\text { la Cámara C, Ventura T, et al. } \\
\text { Revalidación y normalización del Mini- } \\
\text { Examen Cognoscitivo (primera versión } \\
\text { en castellano del Mini-Mental Status } \\
\text { Examination) en la población general } \\
\text { geríátrica. Med Clin-Barcelona. } \\
\text { 1999;112:767-74 } \\
\text { - Blesa R, Pujol M, Aguilar M. Clinical } \\
\text { Validity of the «Mini-Mental State» for } \\
\text { Spanish speaking communities. } \\
\text { Neuropsychologia. 2001;39:1150-7. }\end{array}$ \\
\hline & $\begin{array}{l}\text { Cuestionario portátil } \\
\text { Pfeiffer Versión } \\
\text { Española (SPMSQ-VE) }\end{array}$ & $\begin{array}{l}\text { El Cuestionario de Pfeiffer es otro cuestionario } \\
\text { breve clásico, que valora sobre todo } \\
\text { orientación y también memoria autobiográfica } \\
\text { y cálculo y atención }\end{array}$ & $\begin{array}{l}\text { - Martínez de la Iglesia J, Dueñas } \\
\text { Herrero R, Onís Vilches MC, Aguado } \\
\text { Taberné C, Albert Colomer C, Luque } \\
\text { Luque R. Adaptación y validación al } \\
\text { castellano del cuestionario de Pfeiffer } \\
\text { (SPMSQ) para detectar la existencia de } \\
\text { deterioro cognitivo en personas } \\
\text { mayores de } 65 \text { años. Med Clin (Barc). } \\
\text { 2001;117:129-34 }\end{array}$ \\
\hline & $\begin{array}{l}\text { Test de alteración de la } \\
\text { memoria T@M }\end{array}$ & $\begin{array}{l}\text { El T@M es útil para discriminar el deterioro } \\
\text { cognitivo leve de tipo amnésico y para la } \\
\text { enfermedad de Alzheimer leve. Evalúa } \\
\text { memoria inmediata, semántica, de evocación } \\
\text { libre y con pistas, y orientación temporal }\end{array}$ & $\begin{array}{l}\text { - Rami L, Molinuelo JL, Sánchez-Valle } \\
\text { R, Bosch B, Villar A. Screening for } \\
\text { amnestic mild cognitive imapirment } \\
\text { and early Alzheimer's disease with } \\
\text { M@T (Memory Alteration Test) in the } \\
\text { primary care population. Int J Geriatr } \\
\text { Psychiatry. 2007; 22(4): 294-304 }\end{array}$ \\
\hline
\end{tabular}


Tabla 1 (continuación)

\begin{tabular}{|c|c|c|c|}
\hline Tipología & Instrumento & Descripción & Referencia/s \\
\hline & $\begin{array}{l}\text { Evaluación } \\
\text { cognitiva Montreal } \\
\text { (MoCA). }\end{array}$ & $\begin{array}{l}\text { El MoCA es un instrumento elaborado como } \\
\text { test de cribado del DCL, siendo también } \\
\text { utilizado para la detección de deterioro } \\
\text { cognitivo en diferentes patologías }\end{array}$ & $\begin{array}{l}\text { - Lozano M, Hernández M, Turró O, } \\
\text { Pericot I, López-Pousa S, Vilalta J. } \\
\text { Validación del Montreal Cognitive } \\
\text { Assessment (MOCA): test de cribado } \\
\text { para el deterioro cognitivo leve. Datos } \\
\text { preliminares. Alzheimer Real Invest } \\
\text { Demenc. 2009;43:4-11 }\end{array}$ \\
\hline & $\begin{array}{l}\text { Escala de } \\
\text { demencia de } \\
\text { Blessed }\end{array}$ & $\begin{array}{l}\text { La Escala de demencia de Blessed es utilizada } \\
\text { para cuantificar la capacidad de los pacientes } \\
\text { con demencia para llevar a cabo las tareas de la } \\
\text { vida diaria. Las funciones que evalúa esta escala } \\
\text { son: cambios en la ejecución de las actividades } \\
\text { de la vida diaria, en los hábitos, en la } \\
\text { personalidad y en la conducta. Las puntuaciones } \\
\text { superiores a } 4 \text { indican la presencia de deterioro } \\
\text { cognitivo }\end{array}$ & $\begin{array}{l}\text { - Blessed G, Tomlinson BE, Roth M. } \\
\text { The association between quantitative } \\
\text { measures of dementia and of senile } \\
\text { change in the cerebral grey matter of } \\
\text { elderly subjects. Br J Psychiatry. 1968; } \\
\text { 114(512): 797-811 } \\
\text { - Lozano R, Boada M, Caballero JC, } \\
\text { Flórez F, Garay-Lillo J, González JA. } \\
\text { ABC de las demencias. Barcelona:. } \\
\text { Mayo S.A.; } 1999 \text {. }\end{array}$ \\
\hline \multirow[t]{2}{*}{$\begin{array}{l}\text { Instrumentos de } \\
\text { evaluación de } \\
\text { las demencias }\end{array}$} & ADAS-COG & $\begin{array}{l}\text { Inicialmente diseñada para valorar las } \\
\text { disfunciones características de la EA y que es en } \\
\text { la actualidad la prueba más empleada en } \\
\text { ensayos clínicos y en el seguimiento del } \\
\text { paciente }\end{array}$ & $\begin{array}{l}\text { - Rosen WG, Mohs RC, Davis KL. A } \\
\text { new rating scale for Alzheimer's disease. } \\
\text { Am J Psychiat. 1984;14:1356-64 } \\
\text { - Peña-Casanova J, Aguilar M, Bertran- } \\
\text { Serra I, Santacruz P, Hernández G, Sol } \\
\text { JM. Adaptación y normalización } \\
\text { españolas de la Alzheimer's Disease } \\
\text { Assessment Scale (ADAS) } \\
\text { (NORMACODEM) (y II). Neurología. } \\
\text { 1997;12:69-77 }\end{array}$ \\
\hline & CAMCOG-R & $\begin{array}{l}\text { Desarrollado para determinar presencia, tipo y } \\
\text { severidad de la demencia, permite obtener } \\
\text { puntuaciones de diferentes subescalas en } \\
\text { diferentes áreas del funcionamiento cognitivo y } \\
\text { es sensible al deterioro cognitivo ligero }\end{array}$ & $\begin{array}{l}\text { - Gallagher D1, Mhaolain AN, Coen R, } \\
\text { Walsh C, Kilroy D, Belinski K, et al. } \\
\text { Detecting prodromal Alzheimer's } \\
\text { disease in mild cognitive impairment: } \\
\text { utility of the CAMCOG and other } \\
\text { neuropsychological predictors. Int J } \\
\text { Geriatr Psychiatry. 2010; 25: 1280-7 } \\
\text { - Rodríguez Rodríguez N, Juncos- } \\
\text { Rabadán O, Facal Mayo D. } \\
\text { Discriminación mediante marcadores } \\
\text { cognitivos del deterioro cognitivo leve } \\
\text { frente a envejecimiento normal. Rev Esp } \\
\text { Geriatr Gerontol. 2008;43:291-8. }\end{array}$ \\
\hline
\end{tabular}

Test de Barcelona La versión abreviada del test Barcelona (TBA) abreviado

Clinical Dementia Rating Scale (CDR) intensidad de las demencias

se compone de 55 subpruebas que evalúan la práctica totalidad de ámbitos cognitivos

Ayuda al clínico a estudiar la severidad de la enfermedad de Alzheimer y trastornos asociados en una escala de 0 (normal) a 5 (estadio final), basándose en entrevistas clínicas con un informador y con el paciente. Las áreas que se codifican son memoria, orientación, juicio, resolución de problemas, asuntos comunitarios, hogar y aficiones

Global

Deterioration Scale (GDS)
Se basa en un esquema de progresión del deterioro generalizado y jerárquico. Describe siete estadios globales de demencia, desde la normalidad al deterioro grave. Asume un deterioro progresivo a través de todas las funciones cognitivas, lo que hace que a veces sea difícil de aplicar a pacientes con demencias atípicas
- Peña-Casanova J, Guardia J, BertranSerra I, Manero RM, Jarne A. Versión abreviada del test Barcelona (I): subtests y perfiles normales. Neurología 1997a;12:99-111

- Morris JC. The Clinical Dementia Rating (CDR): current version and scoring rules. Neurology. 1993;43(11):2412-4

- Reisberg B, Ferris SH, de Leon MJ, Crook T. The Global Deterioration Scale for assessment of primary degenerative dementia. Am J Psychiatry. 1982;139(9):1136-9

- Bermejo FP, del Ser T. La graduación de las demencias y su estudio evolutivo. En: Peña-Casanova J, del Ser T eds. Evaluación neuropsicológica y funcional en la demencia. Barcelona: Prous, 1994; 179-200 
Tabla 1 (continuación)

\begin{tabular}{lll}
\hline Tipología & Instrumento & Descripción \\
\hline Functional & Escala utilizada para evaluar el estado funcional y \\
Assessment & determinar el estadio en que se encuentra una \\
Staging (FAST) & demencia. Consta de siete apartados, con \\
& subdivisiones en los apartados seis y siete. Sigue \\
& una escala ordinal desde 1, indicativo de función \\
& normal, a 7, indicativa de demencia grave. La \\
& información obtenida procede de la entrevista con el \\
& cuidador principal o informador fiable. El tiempo de \\
& administración de la prueba oscila entre 15 y 20 \\
& minutos
\end{tabular}

Instrumentos de evaluación del

deterioro

cognitive grave
Severe Cognitive Impairment Profile (SCIP)

Severe Impairment Battery (SIB)

Test for Severe Impairment (TSI)
Instrumentos de evaluación de funciones cognitivas específicas
Específicamente diseñada para los pacientes con demencia grave, con el objetivo de ofrecer información detallada sobre un rango amplio de funciones cognitivas, con una representación adecuada de niveles de dificultad dentro de cada área. Permite a los investigadores producir perfiles neuropsicológicos detallados en pacientes próximo al fallecimiento, permitiendo comparaciones entre la extensión y la severidad de las lesiones neuropatológicas con la severidad de la demencia y los patrones específicos de deterioro cognitivo

Pretende obtener una evaluación neuropsicológica precisa de pacientes con deterioro cognitivo grave. Fue diseñada también para pacientes con demencia grave y tiene en cuenta su comportamiento y déficits cognitivos específicos. Consiste en 40 preguntas que cubren 9 áreas de cognición: interacción social, memoria, orientación, lenguaje, atención, praxias, habilidades visuoespaciales, construcción, y orientación al nombre

Se trata de un test breve que remarca algunas de la preocupaciones planteadas con el MMSE,

minimizando el peso de las habilidades de lenguaje. Fue diseñada para personas con puntuaciones en el MMSE de 10 o menos y cubre 6 áreas: ejecución motora sobreaprendida (psicomotricidad fina y movimientos corporales), comprensión de lenguaje (órdenes simples), producción de lenguaje (denominación), memoria inmediata y diferida (recuerdo libre y reconocimiento), conceptualización (formación de conceptos, cambio y secuenciación, y autoconciencia), y conocimiento general (contar, cantar)

- Test de fluidez verbal

- Test de afasia de Boston
Referencia/s

- Reisberg B. Functional assessment staging (FAST). Psychopharmacol Bull 1988;24:653-9

- Peavy GM, Salmon DP, Rice VA. Neuropsychological assessment of severely demented elderly: the Severe Cognitive Impairment Profile. Arch Neurol. 1996; 53:367-70

- Buiza C. Evaluación y caracterización del deterioro cognitivo grave en personas con diagnóstico de enfermedad de Alzheimer. [Tesis en Internet]. Universidad Autónoma de Madrid. Departamento de Psicología Biológica y de la Salud; 2011 [consultado 30 Ene 2014]. Disponible en:

http://digitooluam.greendata.es:1801/w ebclient/DeliveryManager?pid=39532\& custom att $2=$ simple viewer

- Saxton J, McGonogle-Gibson KL, Swihart AA, Miler VJ, Boller F. Assessment of the severely impaired patient: description and validation of a new neuropsychological test battery.

Psychological assessment. J Consult Clin Psychol. 1990;2(3):298-303

- Llinás Reglá J, Lozano M, López OL, Gudayol M, López-Pousa S, Vilalta J, et al. Validación de la adaptación española de la Severe Impairment Battery (SIB). Neurologia. 1995;10:14-

- Albert MA, Cohen C. The Test for the Severe Impairment: an instrument for the assessment of patients with severe cognitive dysfunction. J Am Geriatr Soc. 1992;40:449-53

- Cuetos-Vega F, Menéndez-González M, Calatayud-Noguera T. Descripción de un nuevo test para la detección precoz de la enfermedad de Alzheimer. Rev Neurol 2007; 44: 469-74

- Goodglass H, Kaplan E. Evaluación de la afasia y de trastornos relacionados. Madrid: Médica Panamericana; 1996 
Tabla 1 (continuación)

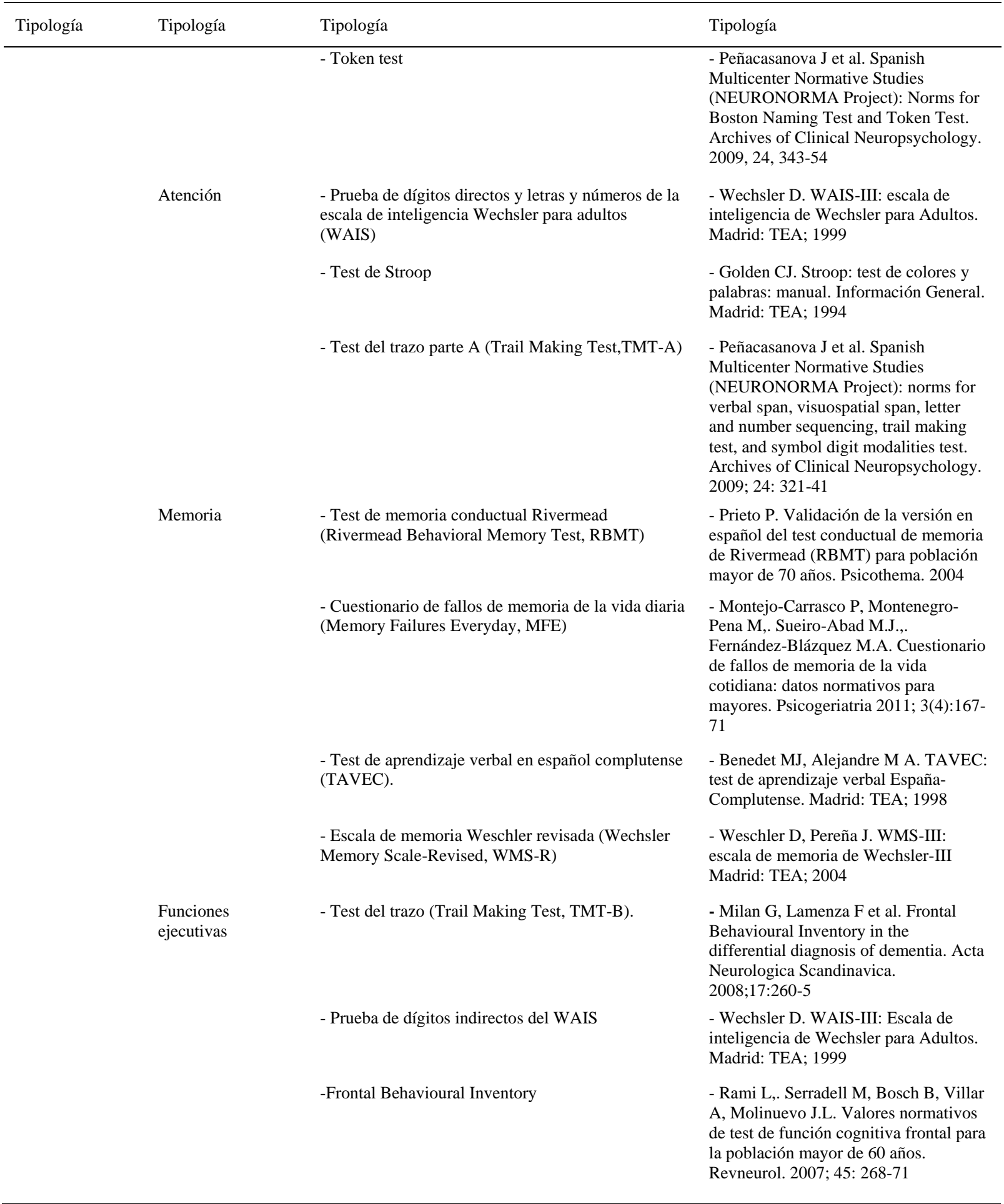

\section{Participantes}

Se obtuvieron 49 respuestas completas al cuestionario, de las cuales $21(42,85 \%)$ eran de profesionales que ejercían su actividad en la provincia de A Coruña, 11 (22,45 \%) en Lugo, 5 (10,21 \%) en Ourense y $12(24,49 \%)$ en Pontevedra. Por perfiles, la mayoría eran profesionales de la psicología $(53,06 \%)$, seguidos de profesionales de la medicina $(20,40 \%)$, terapia ocupacional $(6,12 \%)$ y trabajo social $(6,12 \%)$ y enfermería $(4,08 \%)$. Por ámbitos de trabajo dentro de la atención a las personas mayores, $23(46,94 \%)$ trabajaban en centros de día y $23(46,94 \%)$ en residencias. En este apartado las 
respuestas no eran mutuamente excluyentes, por lo que diferentes profesionales compatibilizaban diferentes dispositivos (por ejemplo, ocho profesionales compatibilizaban el trabajo en centro de día y residencia). De las 49 respuestas, $39(79,6 \%)$ se corresponden a profesionales que aplican directamente la evaluación cognitiva en su trabajo con mayores, a los que por lo tanto el cuestionario les fue aplicado completo (Anexo) y de ellos, 25 informaron haber realizado formación específica sobre evaluación cognitiva y neuropsicológica. La descripción completa puede consultarse en la tabla 2.

$\begin{array}{ll}\text { Tabla } 2 & \\ \text { Participantes en el estudio exploratorio sobre el uso de instrumentos de } \\ \text { evaluación cognitiva y neuropsicológica en Galicia, según la provincia, el } \\ \text { perfil profesional y tipo de centro }\end{array}$

\section{Resultados}

\section{Información cuantitativa}

De los 49 profesionales que completaron la escala, el $2 \%$ considera muy poco importante la evaluación cognitiva (un participante), el $12 \%$ poco importante (6 participantes), el $8 \%$ algo importante (4 participantes), el $37 \%$ importante (18 participantes) y el $41 \%$ muy importante (20 participantes).

La figura 1 muestra la frecuencia de utilización de test de cribado, escalas globales de demencia, baterías de evaluación neuropsicológica generales y baterías de evaluación del deterioro cognitivo severo en los 39 participantes del estudio que aplicaban estimulación cognitiva en sus centros. En cuanto a los test de cribado, todos los participantes emplean diferentes versiones del MMSE. De las diferentes versiones disponibles en castellano del MMSE, la versión más frecuente es la de Lobo et al ${ }^{10}$, que informan emplear 27 participantes, seguida de la versión NORMACODEM ${ }^{11}$, en 4 participantes. Además de los tests incluidos en el cuestionario, 9 participantes emplean el test del reloj con fines de detección del deterioro cognitivo, 3 participantes emplean el Fototest ${ }^{12}$ y 2 el Memory Impairment Screen ${ }^{13}$. En cuanto a las baterías globales de demencia, una mayoría de los participantes emplean el GDS como instrumento de estadificación, seguido por el CDR y el FAST. Tres de los participantes emplean como instrumento de estadificación el VIRO, un test de orientación con carácter global ${ }^{14}$. En cuanto a las baterías generales de evaluación neuropsicológica, los instrumentos más utilizados son el CAMCOG-R y el ADAS-COG, seguidos por el Test Barcelona abreviado. Dos participantes indican en esta sección aplicar subtests del Test Barcelona. Respecto a las baterías para la evaluación del deterioro cognitivo severo, el instrumento más utilizado es el Severe Impairment Battery, seguido del Test for Severe Impairment y el Severe Cognitive Impairment Profile. Dos participantes indican utilizar como instrumento de evaluación el $\mathrm{SMMSE}^{15}$, que si bien se trata de un instrumento de cribado más que de evaluación completa, sí es específico de este grupo de deterioro. 

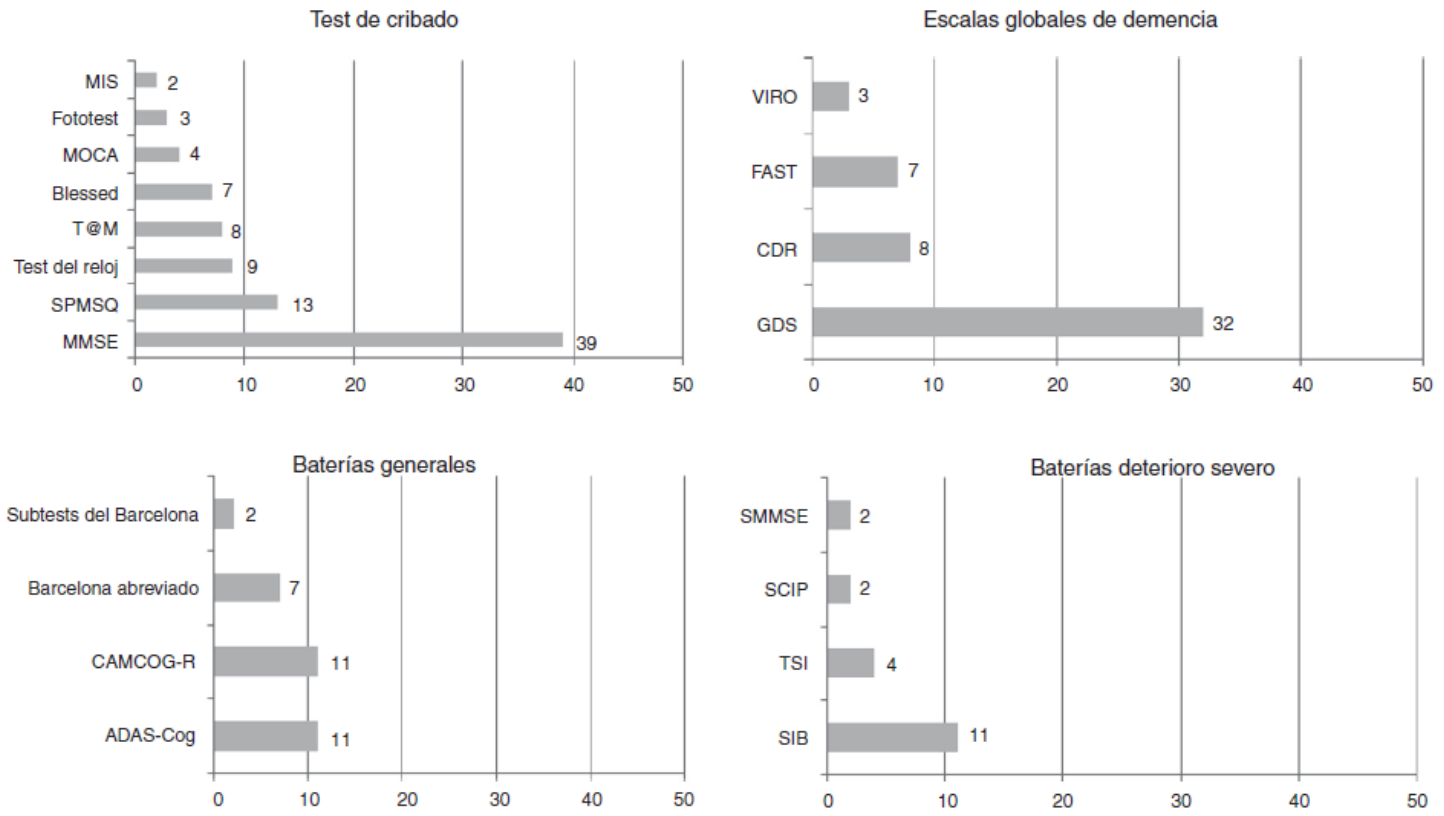

Figura 1. Frecuencia en el uso test de cribado, escalas globales de demencia, baterías generales y baterías de deterioro cognitivo severo en una muestra no representativa de profesionales gallegos. ADAS-cog: Alzheimer's Disease Assessment Scale-cognitive subscale; CAMCOG-R: Cambridge Cognitive Examination-Revised; CDR: Clinical Dementia Rating Scale; FAST: Functional Assessment Staging; GDS: Global Deterioration Scale; MIS: Memory Impariment Screen; MMSE: Mini - Mental State Examination; MOCA: Montreal Cognitive Assessment; SCIP: Severe Cognitive Impairment Profile; SIB: Severe Impairment Battery; SMMSE: Severe Mini Mental State Examination; SPMSQ: Short Portable Mental Status Questionnaire; TSI: Test for Severe ImpairmentT@M: Test de Alteración de la Memoria.

Respecto a test específicos para determinadas funciones cognitivas, 11 participantes informan emplear test de memoria ( 3 test de aprendizaje de listas de palabras, 2 test Rivermead, 6 otros), 9 test de lenguaje (3 Token Test, 2 test de fluidez, 2 Boston abreviado, 2 test de lenguaje expresivo Kerst, uno test de denominación de Boston, uno set test), 7 test de atención (3 Trail Making Test parte A, 3 test de Stroop, uno test de cancelación del WAIS, uno CAM), 6 test de funciones ejecutivas ( 3 Trail Making Test parte B, uno Frontal Behavioural Inventory, uno pruebas de resolución de problemas, uno pruebas de amplitud de memoria de trabajo), 2 tests de praxias y uno test de gnosias.

\section{Información cualitativa}

A través de preguntas abiertas, se han recogido las opiniones de los participantes sobre la evaluación cognitiva en centros, las cuales se han estudiado a través de un análisis de contenidos. Profundizando en las diferentes limitaciones descritas por cada uno de los 49 participantes, se ha optado por agruparlas en 3 grandes bloques para una mejor comprensión de las opiniones declaradas: 1) limitaciones relacionadas con la persona evaluada, 2) con la persona evaluadora o el centro de trabajo y 3) con las trabas surgidas de los propios instrumentos de valoración. Del total de comentarios, un 45,09\% hace referencia al primer tipo, un 33,33 \% al segundo y un 21,56 \% al tercero. Entre las limitaciones relacionadas con la persona evaluada, destacan las referidas a aspectos sociodemográficos, incluyendo el bajo nivel educativo [«La principal limitación es el grado de deterioro del anciano que llega al centro y en ocasiones el bajo nivel cultural», 20 a 35 años, psicología, residencia]. También se señala el deterioro cognitivo avanzado y la presencia de otras patologías, déficits o trastornos (trastorno psiquiátrico, polifarmacia, trastorno del lenguaje, déficit sensorial, etc.) como aspectos que dificultan o interfieren en la valoración de las funciones cognitivas. Con frecuencia se advierte como un inconveniente relacionado con el profesional o el centro de trabajo, la falta de tiempo para la realización de valoraciones objetivas exhaustivas [«El protocolo de valoraciones es el que marca qué pruebas debo aplicar. Es cierto que a mayores de las que he descrito puedo aplicar las que yo desee pero la limitación más importante es el tiempo que se me asigna en los horarios para realizar las valoraciones», 20 a 35 años, psicología, residencia]. También la carencia de recursos materiales o humanos (insuficiente formación del profesional) y la escasa conciencia del valor y la utilidad de la evaluación cognitiva son algunas de las restricciones señaladas por los participantes. En cuanto a las limitaciones que plantean los instrumentos de evaluación, los participantes subrayan la ausencia de valores normativos y baremos adaptados a la realidad de la población evaluada [«Las pruebas 
de screening son demasiado generales y las pruebas específicas están muy influenciadas por el nivel cultural. No hay escalas adaptadas a nuestra población», 35 a 50 años, psicología, centro de día]. En concreto, de los comentarios referidos a las limitaciones de la persona evaluada, un 47,82 \% hacen referencia a aspectos sociodemográficos, un 26,08 \% al nivel de deterioro y un $26,08 \%$ a la presencia de otras patologías; de los referidos a las limitaciones de la persona evaluadora o del centro, un 64,70\% se refieren a la falta de tiempo, un 17,64 \% a la falta de recursos y un 17,64\% a la escasa conciencia del valor de la evaluación cognitiva; mientras que los comentarios referidos a las limitaciones de los propios instrumentos de valoración hacen referencia a la ausencia de baremos y valores normativos.

De las opiniones libres, y comentarios vertidos por los profesionales, también se extraen ideas que, en análisis, nos acercan a una concepción más detallada de su labor diaria y de las formas empleadas para hacer frente a los retos cotidianos que conllevan las limitaciones citadas anteriormente. Así por ejemplo, se pone de relieve la utilidad de la observación rutinaria en el entorno cotidiano de la persona evaluada, como herramienta compensatoria - y a veces incluso sustitutiva-, y no complementaria, ante la falta de tiempo disponible para las evaluaciones cognitivas [«Con el MMSE evito aplicar una prueba potencialmente más estresante para demostrar algo que ya sé por simple observación», 20 a 35 años, psicología, centro de día]. Algunos reconocen verse obligados a emplear únicamente pruebas de cribado para hacer frente a sus retos cotidianos [«En la práctica diaria suelo utilizar test de screening y escalas globales de demencias, principalmente por disponibilidad de tiempo. Solo para el estudio de casos puntuales utilizo baterías neuropsicológicas y test específicos», 20 a 35 años, psicología, centro de día y residencia]. Por otro parte, se destaca la importancia de los seguimientos en las valoraciones cognitivas [ «Su relevancia es solo para un conocimiento inicial del usuario, es decir, para ver cómo entra y poco más. Apenas hay seguimiento ni trabajo de refuerzo o mantenimiento de las capacidades que poseen», 35 a 50 años, auxiliar, residencia] no solo como un trámite de obligado cumplimiento, sino también como una herramienta para conocer el desarrollo de la persona evaluada y la eficacia de los programas de intervención. Además, los participantes demandan la adaptación y/o creación de instrumentos de valoración cognitiva [«Las pruebas cognitivas se ven sesgadas por el nivel cultural del usuario en muchas ocasiones, haciendo necesario cruzar varias escalas o incluso basarse en la observación», 20 a 35 años, trabajo social, residencia] y destacan la necesidad de que estos instrumentos se adecúen a su día a día profesional (tiempo, disponibilidad de materiales, etc.), así como a los perfiles de los evaluados (deterioro avanzado, bajo nivel de escolarización, etc.). En los comentarios de los participantes, también queda patente la necesidad de implicación de todo el equipo multidisciplinar para una completa y ajustada valoración cognitiva (psicología, medicina, logopedia, terapia ocupacional, auxiliares, etc.) [《Los médicos deberíamos usar más frecuentemente la evaluación cognitiva de nuestros pacientes», más de 50 años, médico, residencia]. Algunos participantes demandan cursos, jornadas y foros formativos de calidad que permitan compartir, adquirir y actualizar conocimientos de evaluación cognitiva, desde una perspectiva realista y que tenga en cuenta las exigencias de las limitaciones diarias.

\section{Discusión}

Los resultados sobre el uso de test cognitivos y neuropsicológicos de Galicia muestran una alta aplicación del MMSE como herramienta de cribado o aplicación breve, y del GDS como escala global de demencia. Estos resultados podrían explicarse, al menos parcialmente, por la existencia en los centros de protocolos de evaluación cerrados y por el elevado grado de deterioro que existe en los centros, que dificulta la aplicación de pruebas más complejas. En el campo de las baterías específicas de demencia, la variabilidad es mayor y parece reflejar un mayor grado de reflexión y elaboración por parte de los profesionales. La inclusión en estas baterías de tareas o ítems específicos para determinadas funciones cognitivas, o incluso de subescalas para determinadas áreas como en el caso del CAMCOG-R, podría explicar parcialmente la baja utilización de pruebas específicas. La diversidad de resultados se justifica en la diversidad del sector, con diferentes servicios en diferentes áreas de atención (clínica, social, asistencial). En este sentido, aunque exploratorio, el presente estudio parece indicar un riesgo de polarización del sector, en el que un porcentaje elevado de profesionales emplearía unos pocos instrumentos con objetivos limitados, y un porcentaje pequeño de profesionales emplearía un número elevado de instrumentos específicos, pero cuya utilidad se ve restringida por no ser un uso compartido con el resto del equipo y/o el sector.

Además de limitaciones ya conocidas en la aplicación de test de evaluación cognitiva en centros de personas mayores (p.ej. alto grado de deterioro de los usuarios; escasez de tiempo y organización del equipo multidisciplinar), emergen del presente estudio una serie de temas relevantes para el desempeño profesional. Por un lado, la diversidad de pruebas empleadas, así como las reticencias de los profesionales respecto a los instrumentos disponibles, señala la necesidad de un consenso en evaluación cognitiva y neuropsicológica en centros de personas mayores. Este consenso debería facilitar el desempeño de los 
profesionales, objetivar sus necesidades formativas y mejorar, en relación a los usuarios, tanto la valoración cognitiva como la necesaria interrelación entre evaluación e intervención. La nueva configuración de los servicios de la atención a la dependencia en Galicia, concretada en la cartera de servicios de la Consellería de Benestar de la Xunta de Galicia ${ }^{16}$, subraya la necesidad de emplear la información recogida a través de la evaluación en la preparación de planes de atención individualizados realmente personalizados y adaptados a las necesidades funcionales y cognitivas. Así mismo, los resultados recogidos destacan el papel de la observación como complemento de la evaluación cognitiva e instrumento de evaluación de la eficacia de la intervención, el cual no obstante debe ser consolidado a través del desarrollo de instrumentos metodológicamente robustos y empíricamente validados ${ }^{17}$.

El presente estudio exploratorio sirve también para poner el foco en la necesidad de disponer de instrumentos sensibles al cambio y aprovechar las evaluaciones repetidas requeridas en los centros de personas mayores dependientes para mejorar nuestro conocimiento sobre el deterioro cognitivo y la efectividad de las intervenciones aplicadas ${ }^{3,9}$. Los instrumentos de evaluación cognitiva y neuropsicológica debieran emplearse para el diagnóstico continuado y, fundamentalmente, para la elaboración de planes de intervención. En los centros de mayores, discapacidad y dependencia de Galicia la valoración de la persona usuaria por parte de los profesionales se realiza en la primera semana de ingreso, debiendo completarse el plan de atención individualizada en el primer mes y ser revisado como mínimo anualmente o siempre que exista una variación significativa en la situación ${ }^{18}$. Así, el presente estudio debiera favorecer la deliberación sobre la necesidad del empleo de instrumentos no solo para tipificar o etiquetar sino para realizar un seguimiento longitudinal, aprovechando las sinergias surgidas en los centros de la aplicación de la normativa que determina al menos una evaluación anual de la persona usuaria. Esta necesidad se justifica tanto por la presencia de comentarios cualitativos de los profesionales sobre la importancia del seguimiento como por la ausencia entre los test más utilizados de pruebas sensibles al cambio o que, simplemente, dispongan de versiones paralelas.

Este estudio presenta diferentes limitaciones, asociadas al carácter exploratorio del mismo y al tamaño de la muestra, que compromete la generalización de los resultados. Una recogida de datos de tipo cuantitativo más generalizada y un análisis más pormenorizado de los mismos permitirán establecer perfiles de uso de los test en función de la tipología profesional, el entorno de trabajo e incluso las características sociodemográficas de los profesionales. Así mismo, una recogida más detallada de datos de tipo cualitativo permitirá avanzar en un consenso sobre evaluación de las funciones cognitivas y su impacto en los servicios y cuidados prestados a las personas mayores. En este sentido, el objetivo del grupo de trabajo de la SGXX es la elaboración de protocolos de evaluación cognitiva asumibles por un amplio rango de profesionales y centros, y aplicables satisfactoriamente a lo largo del tiempo. 
Anexo

Estimado/a socio/a:

El presente cuestionario ha sido elaborado por el grupo de trabajo sobre evaluación cognitiva y neuropsicológica de la Sociedad Gallega de Gerontología y Geriatría.

Nuestro objetivo es recabar información sobre la evaluación cognitiva y neuropsicológica en los centros de personas mayores de Galicia. Como profesional de la gerontología y la geriatría, sus respuestas nos son relevantes, tanto si usted aplica este tipo de evaluación directamente como si otros profesionales lo hacen en su centro.

Se trata de un breve cuestionario, que cumplimentarlo no le llevará más de 5 minutos. Por favor, lea cuidadosamente las preguntas y conteste con sinceridad.

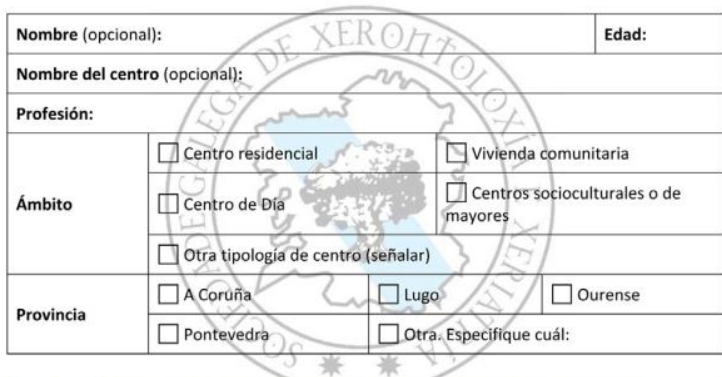

\begin{tabular}{|c|l|}
\hline 1 & $\begin{array}{l}\text { En su centro de trabajo, ¿usted aplica pruebas de evaluación cognitiva a } \\
\text { personas mayores? }\end{array}$ \\
$\square$ No & $\begin{array}{l}\text { 1a. RESPUESTA NEGATIVA A LA PREGUNTA 1 } \\
\text { ¿Usted conoce si en su centro se aplican pruebas de evaluación cognitiva a } \\
\text { personas mayores? : } \square \text { NO } \square \text { sI } \\
\text { Caso afirmativo: ¿Qué profesional las aplica? }\end{array}$ \\
\hline Si & $\begin{array}{l}\text { 21. RESPUESTA AFIRMATIVA A LA PREGUNTA 1: } \\
\text { ¿Cuáles son las pruebas de screening o despistaje que aplica? } \\
\square \text { MMSE. En caso afirmativo, ¿́qué versión está utilizando? }\end{array}$ \\
\hline $\begin{array}{l}\text { Test de Pfeiffer (SPMSQ) } \\
\text { Test de Alteración de la Memoria (T@M) } \\
\square \text { Test de Evaluación Cognitiva Montreal (MOCA) } \\
\square \text { Test de demencia de Blessed }\end{array}$ \\
\hline
\end{tabular}

Sociedade Galega de Xerontoloxia E Xeriatria
GRUPO TRABAJO

PSICOGERONTOLOGÍA

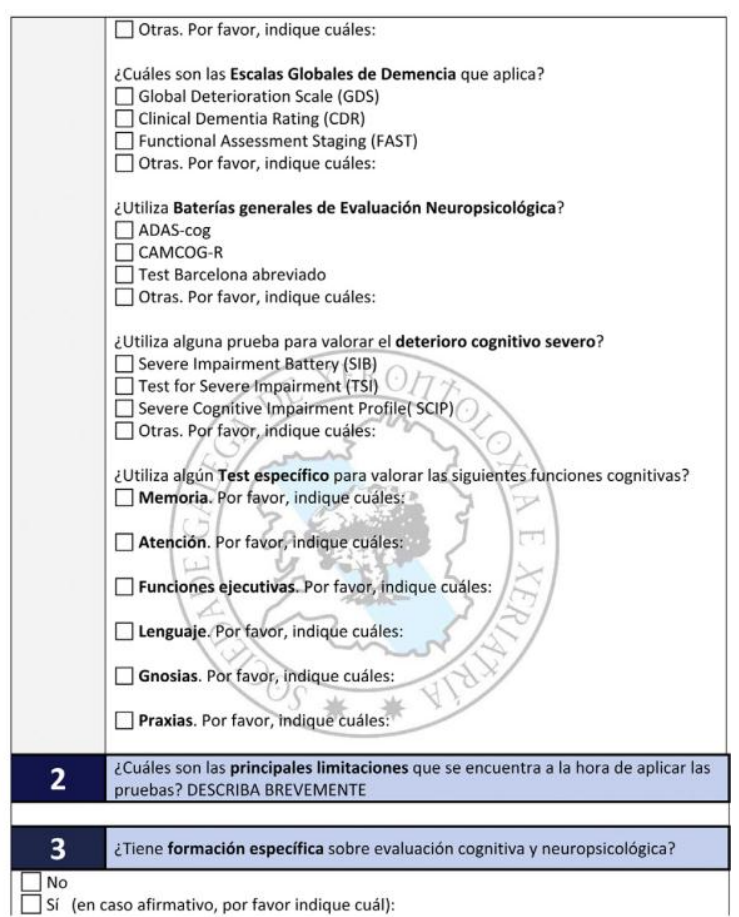

Si (en caso afirmativo, por favor indique cuál):

Sociedade Galega de Xerontoloxiae Xeriatria

GRUPO TRABAJO

4 De 1 a 5, ¿Qué importancia tiene en el funcionamiento de su centro de trabajo evaluación cognitiva de las personas usuarias?

\begin{tabular}{|c|c|c|c|c|c|c|}
\hline $\begin{array}{c}\text { Muy poco } \\
\text { importante }\end{array}$ & 1 & 2 & 3 & 4 & 5 & $\begin{array}{c}\text { Muy } \\
\text { importan }\end{array}$ \\
\hline
\end{tabular}

Por favor, a continuación haga cualquier comentario que considere pertinente en relación al tema del cuestionario

5 Otras recomendaciones

MUCHAS GRACIAS POR SU COLABORACIÓN.

LA SOCIEDAD GALLEGA DE GERONTOLOGIA Y GERIATRÍA LE MANTENDRÁ INFORMADO/A DE IOS RESULTADOS OBTENIDOS POR ESTE GRUPO DE TRABAIO

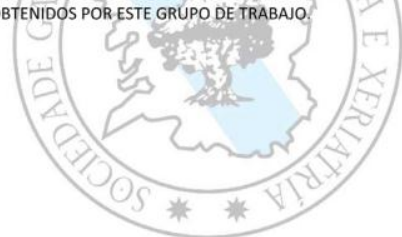




\section{Bibliografía}

1. Lee MM, Camp CJ. El papel de la evaluación cognitiva en el tratamiento de la demencia avanzada. Rev Esp Geriatr Gerontol. 2011:46, http://dx.doi.org/10.1016/j.regg.2011.02.001.

2. Cliffe S, Robinson L, Brayne C, Goodman C, Rait G, Manthorpe J, et al. Primary care and dementia: 1. Diagnosis, screening and disclosure. Int J Geriatr Psychiatry. 2009;24:895-901.

3. Facal D, Guàrdia-Olmos J, Juncos-Rabadán O. Aportaciones metodológicas al estudio de datos de tipo longitudinal en el deterioro cognitivo ligero. Rev Esp Geriatr Gerontol. 2014, http://dx.doi.org/10.1016/j.regg.2011.02.001.

4. Villarejo A, Puertas-Martin V. Utilidad de los test breves en el cribado de demencia. Neurología. 2011;26:425-33.

5. Delgado D, Guerrero Bonnet S, Troncoso Ponce M, Araneda Yáñez A, Slachevsky Chonchol A, Behrens Pellegrino MI. Memoria, fluidez y orientación: prueba de cribado de deterioro cognitivo en 5 minutos. Neurología. 2013;28:400-7.

6. Peña-Casanova J, Gramunt NF, Gich JF. Test neuropsicológicos. En: Fundamentos para una psicología clínica basada en evidencias. Barcelona: Masson; 2004.

7. Reisberg B, Schneider L, Doody R, Anand R, Feldman H, Haraguchi H, et al. Clinical global measures of dementia position paper from the International Working Group on Harmonization of Dementia Drug Guidelines. Alzheimer Dis Assoc Disord. 1997;11 Suppl 3:8-18.

8. Lezak M. Neuropsychological Assessment. 3.a ed. Nueva York: Oxford University Press; 1996.

9. Balo García A. Evaluación y seguimiento del estado cognitivo y afectivo de una muestra de personas mayores institucionalizadas. Tesis doctoral, Universidade da Coruña; 2013.

10. Lobo A, Saz P, Marcos G, Día JL, de la Cámara C, Ventura T, et al. Revalidación y normalización del Mini-Examen Cognoscitivo (primera versión en castellano del Mini-Mental Status Examination) en la población general geríátrica. Med ClinBarcelona. 1999;112:767-74.

11. Blesa R, Pujol M, Aguilar M. Clinical Validity of the «Mini-Mental State» for Spanish speaking communities. Neuropsychologia. 2001;39: 1150-7.

12. Carnero- Pardo C, Montoro-Ríos MT. Test de las fotos. Rev Neurol. 2004;39:801-6.

13. Bushcke H, Kurlansky G, Katz M. Screening for dementia with the Memory Impairment Screen. Neurology. 1999;112:231-8.

14. Kastenbaum R, Sherwood S. VIRO: A scale for assessing interview behaviours of elderly people. En: Kastenbaum KD, editor. Research, planning and action for the elderly. New York: Behavioural Publications; 1972.

15. Buiza C, Navarro A, Díaz-Orueta U, González MF, Álaba J, Arriola E, et al. Evaluación breve del estado cognitivo de la demencia en estadios avanzados: resultados preliminares de la validación española del Severe Mini-Mental State Examination. Rev Esp Geriatr Gerontol. 2011;46:131-8.

16. Consellería de Traballo e Benestar Social. Cartera de servicios para personas dependientes, prevención de la dependencia y promoción de la autonomía personal. Santiago de Compostela: Xunta de Galicia; 2011.

17. García-Soler A, Buiza Bueno C, Vaca Bermejo R, Ansorena Urchegui X. Desarrollo de un instrumento de registro de implicación/engagement en la actividad para la observación sistemática de personas mayores con deterioro cognitivo. Rev Esp Geriatr Gerontol. 2014;49:65-8.

18. Consellería de Traballo e Benestar Social. Protocolo da inspección de servizos sociais sobre os centros de maiores, discapacidade e dependencia da comunidade autónoma de Galicia. Santiago de Compostela: Xunta de Galicia; 2011 [consultado 2 Jun 2014]. Disponible en: http://avepss.traballoebenestar.xunta.es/documentacion/protocolo inspeccion maiores discapacidade dependencia.Pdf 